\title{
Heat capacity and thermodynamic properties of strontium from 5 to $350 \mathrm{~K}^{\text {s }}$
}

\author{
JULIANA BOERIO and EDGAR F. WESTRUM, JR. \\ Department of Chemistry, University of Michigan, Ann Arbor, \\ Michigan 48109, U.S.A.
}

(Received 23 February 1977)

\begin{abstract}
The heat capacity of pure strontium metal was measured by adiabatic-shield calorimetry from 5 to $350 \mathrm{~K}$. At $298.15 \mathrm{~K}$ values of the thermodynamic functions are found to be: $C_{\mathrm{p}} / \mathrm{cal}_{\mathrm{th}} \mathrm{K}^{-1} \mathrm{~mol}^{-1}=6.402, S^{\circ} / \mathrm{cal}_{\mathrm{th}} \mathrm{K}^{-1} \mathrm{~mol}^{-1}=13.313,\left\{H(T)-H^{\circ}(0)\right\} / \mathrm{cal}_{\mathrm{th}} \mathrm{mol}^{-1}$ $=1570.2$, and $\left\{G^{\circ}(T)-H^{\circ}(0)\right\} / T \mathrm{cal}_{\mathrm{tn}} \mathrm{K}^{-1} \mathrm{~mol}^{-1}=8.047$. Although the entropy at this temperature is in reasonable accord with another measurement $\left(13.24 \mathrm{cal}_{\mathrm{ch}} \mathrm{K}^{-1} \mathrm{~mol}^{-1}\right)$ reported in the literature since initiation of these studies, serious differences in the temperature dependence of the heat capacity can be best explained by the assumption that the other sample contained approximately 10 moles per cent of strontium oxide.
\end{abstract}

\section{Introduction}

When plans for these adiabatic heat-capacity measurements on strontium were made at the behest of the CODATA Task Group on Key Values for Thermodynamic Properties, experimental thermodynamic data on this metallic element were scarce indeed. (Quite possibly it was not so much a matter of being overlooked-as avoided!) Only estimates ${ }^{(1,2)}$ of the entropy of this important element were available at $300 \mathrm{~K}$. These estimates were based primarily on a single study ${ }^{(3)}$ of the heat capacity of strontium between 1.5 and $20 \mathrm{~K}$, but a crude, enthalpy-type measurement did in fact exist at $300 \mathrm{~K} \cdot{ }^{(4)}$ Since the initiation of these experiments, a more comprehensive study has been undertaken elsewhere ${ }^{(5,6)}$ which gave an entropy at $298.15 \mathrm{~K}$ in reasonable accord with that reported here. However, serious differences in the heat capacities of that study ${ }^{(5,6)}$ and those of the present measurements exist over the common temperature range of measurements. These differences can most consistently be interpreted on the assumption that their sample contained approximately 10 moles per cent of strontium monoxide as a contaminant. We consider that the heat-capacity and derived thermodynamic values from 5 to $350 \mathrm{~K}$ reported in this paper are the most reliable.

\section{Experimental}

\section{SAMPLE PREPARATION AND HANDLING}

The high-purity strontium metal sample was kindly provided through the courtesy of Dr David T. Peterson of the Ames Laboratory of the Energy Research and

a This work was supported in part at the University of Michigan by the Chemical Thermodynamics Program, Chemistry Section, National Science Foundation under contract no. GP $-42525 X$. 
Development Administration located at Iowa State University, Ames, Iowa. It was prepared by fractionally subliming the best commercial-grade strontium available from King Laboratories. The first fraction consisting of roughly 10 per cent of the original starting material was relatively high in magnesium and was rejected. The middle fraction consisting of approximately 65 per cent of the original was purest; it alone was further purified. The residue of about 25 per cent was rich in calcium and barium. After resublimation of the middle fraction, it was sealed in a tantalum container in an inert atmosphere and maintained for $5 \mathrm{~d}$ at a temperature of $1200 \mathrm{~K}$ under high vacuum to permit hydrogen to diffuse from the sample. Careful analytical study of the sample indicated the following mass percentages ascertained by the method indicated in parentheses. With the exception of the vacuum fusion done at Ames, all of the analyses were made through the courtesy of the Analytical Group of the CMB Division of the Los Alamos Scientific Laboratory, University of California, Los Alamos, New Mexico. H, 0.0024 (vacuum fusion); ${ }^{(7)} \mathrm{H}, 0.0010$ (LECO hydrogen analyzer); $\mathrm{O},<0.010$ (neutron activation analysis); $\mathrm{N}, 0.0012$ (chemical analysis); C, 0.0040 (LECO low-carbon analyzer); $\mathrm{Ca}, 0.0423$ (atomic absorption); and $\mathrm{Ba}, 0.0615$ (atomic absorption). Since strontium is readily oxidized, the sample was handled exclusively in a drybox containing a purified anhydrousnitrogen atmosphere.

\section{CALORIMETER AND CRYOSTAT}

The $47.349 \mathrm{~g}$ strontium sample in the form of pellets with typical dimensions of several $\mathrm{mm}$ was loaded into the gold-plated OFHC (oxygen-free high-conductivity) copper calorimeter (laboratory designation W-54) provided with a screw-type closure involving a stainless-steel knife edge and an annealed gold gasket. The loaded calorimeter was removed from the drybox in a stainless-steel "loading chamber", evacuated, and after addition of $2.53 \mathrm{kPa}$ at $300 \mathrm{~K}$ of purified helium to facilitate thermal equilibration, the calorimeter was sealed. A measured amount of Apiezon-T grease was placed in the thermocouple and heater-thermometer wells of the calorimeter to provide good thermal contact between the heater, the temperature sensors, and the calorimeter. The added quantities of helium and grease match those used during the measurement of the heat capacity of the empty calorimeter. The fraction of the total heat capacity (calorimeter + sample) due to the sample decreased from 0.89 at $10 \mathrm{~K}$ to 0.49 at $100 \mathrm{~K}$ and to 0.42 at $350 \mathrm{~K}$.

The low-temperature heat-capacity measurements were then made in the Mark-II adiabatic cryostat described elsewhere. ${ }^{(8)}$ A capsule-type platinum resistance thermometer (laboratory designation A-5) calibrated at the U.S. National Bureau of Standards was used for temperature and temperature-increment determinations. Measurements of other quantities involved in the calorimetric experiment, such as mass, current, potential, and time were also referenced to calibrations or standards of that laboratory.

The accuracy of a single measurement in this endeavor is judged to be about 5 per cent at $10 \mathrm{~K}$ decreasing to 0.1 per cent at $25 \mathrm{~K}$ to about 0.06 per cent between 25 and $350 \mathrm{~K}$ even though precision is significantly better at low temperatures. Insofar as the smoothed functions are concerned, the heat capacity above $100 \mathrm{~K}$ is 
precise to \pm 0.1 per cent and the thermodynamic functions to about 0.06 per cent in terms of the standard deviation as the precision index.

\section{Results}

The heat capacity of strontium may be represented by a simple sigmate curve from 5 to $350 \mathrm{~K}$. The experimental heat capacities (table 1, based on a molar mass of $87.62 \mathrm{~g} \mathrm{~mol}^{-1}$ for $\mathrm{Sr}$ ) have been adjusted for curvature to correct for the difference

TABLE 1. Experimental heat capacity of strontium $\left(\mathrm{cal}_{\mathrm{th}}=4.184 \mathrm{~J}\right)$

\begin{tabular}{|c|c|c|c|c|c|c|c|}
\hline$T$ & $C_{p}$ & $T$ & $C_{p}$ & $T$ & $C_{p}$ & $\underline{T}$ & $C_{p}$ \\
\hline $\overrightarrow{\mathbf{K}}$ & $\mathrm{cal}_{\mathrm{th}} \mathrm{K}^{-1} \mathrm{~mol}^{-1}$ & $\vec{K}$ & $\mathrm{cal}_{\mathrm{th}} \mathrm{K}^{-1} \mathrm{~mol}^{-1}$ & $\mathrm{~K}$ & $\mathrm{cal}_{\mathrm{th}} \mathrm{K}^{-1} \mathrm{~mol}^{-1}$ & $\bar{K} \mathbf{c}$ & $\mathrm{cal}_{\mathrm{th}} \mathrm{K}^{-1} \mathrm{~mol}^{-1}$ \\
\hline \multicolumn{2}{|r|}{ Series I } & \multicolumn{2}{|r|}{ Series III } & \multicolumn{2}{|r|}{ Series IV } & \multicolumn{2}{|r|}{ Series V } \\
\hline 50.09 & $4.464^{a}$ & 192.14 & . $\quad 6.131$ & 10.81 & 0.292 & 117.40 & 5.770 \\
\hline 53.20 & 4.516 & 202.36 & 6.152 & 12.06 & 0.383 & 127.77 & 5.854 \\
\hline 58.55 & 4.741 & 212.48 & 6.179 & 13.38 & 0.505 & 128.10 & 5.918 \\
\hline 64.55 & 4.962 & 222.52 & 6.224 & 14.79 & 0.674 & 148.33 & 5.975 \\
\hline 71.77 & $5.183^{a}$ & 232.49 & 6.247 & 16.38 & 0.857 & 158.50 & 6.016 \\
\hline 79.55 & 5.317 & 242.65 & 6.272 & 18.07 & 1.087 & 168.67 & 6.066 \\
\hline 88.47 & 5.484 & 253.01 & 6.308 & 19.92 & 1.347 & 178.86 & 6.085 \\
\hline 99.16 & 5.607 & 263.58 & 6.339 & 22.01 & 1.636 & 189.12 & 6.110 \\
\hline \multirow{2}{*}{110.68} & 5.716 & 274.09 & 6.364 & 24.32 & 1.966 & 199.43 & 6.130 \\
\hline & & 284.68 & 6.386 & 26.85 & 2.299 & 209.61 & 6.161 \\
\hline \multirow{2}{*}{\multicolumn{2}{|c|}{ Series II }} & 295.33 & $6.421^{a}$ & 29.60 & 2.640 & 219.84 & 6.206 \\
\hline & & 305.75 & 6.416 & 32.66 & 2.997 & 230.12 & 6.218 \\
\hline 112.25 & 5.734 & 316.13 & 6.425 & 35.96 & 3.335 & 240.43 & 6.265 \\
\hline 122.73 & 5.826 & 326.58 & $6.476^{a}$ & 39.52 & 3.656 & 250.76 & 6.288 \\
\hline 132.88 & 5.890 & 337.03 & $6.505^{a}$ & 43.64 & 3.967 & 261.02 & 6.318 \\
\hline 143.18 & 5.955 & 346.42 & $6.498^{a}$ & 48.31 & 4.265 & 271.22 & 6.354 \\
\hline 153.68 & 6.003 & \multirow{2}{*}{\multicolumn{2}{|c|}{ Series IV }} & 52.47 & 4.482 & 281.52 & 6.380 \\
\hline 164.00 & 6.040 & & & 57.20 & 4.687 & 291.91 & 6.396 \\
\hline 174.35 & 6.080 & 6.28 & 0.067 & 63.61 & 4.926 & 302.28 & 6.404 \\
\hline 184.77 & 6.106 & $\begin{array}{l}6.20 \\
6.82\end{array}$ & 0.083 & 70.74 & 5.114 & 312.61 & 6.424 \\
\hline \multirow{2}{*}{\multicolumn{2}{|c|}{ Series III }} & 7.65 & 0.110 & \multirow{2}{*}{\multicolumn{2}{|c|}{ Series V }} & 322.91 & 6.433 \\
\hline & & 8.61 & 0.148 & & & 333.19 & 6.447 \\
\hline 182.13 & $6.166^{a}$ & 9.67 & 0.203 & 106.90 & 5.679 & 343.42 & 6.466 \\
\hline
\end{tabular}

${ }^{a}$ Omitted from curve fit.

between the experimentally measured $\Delta H / \Delta T$ and the value obtained in the limit as the temperature increment approaches zero. At the lowest temperatures the heat capacity of strontium may be fitted conveniently to an equation of the form: $C_{p}=\alpha T^{3}+\beta T$. Here the cubic term in $T$ represents the lattice contribution of total heat capacity and that linear in $T$ is the conduction-electron contribution. Values for $\alpha$ and $\beta$ were obtained by a least-squares fitted curve through the results below $10 \mathrm{~K}$ to the rectified plot of $C_{p} / T$ against $T^{2}$. The coefficients are found to be $\alpha=1.99 \times 10^{-4} \mathrm{cal}_{\mathrm{th}} \mathrm{K}^{-4} \mathrm{~mol}^{-1}$ and $\beta=3.3 \times 10^{-3} \mathrm{cal}_{\mathrm{th}} \mathrm{K}^{-2} \mathrm{~mol}^{-1}$. $\dagger$ The results over the entire temperature range were fitted to a polynomial in temperature, again

$\dagger$ Throughout this paper $\mathrm{cal}_{\mathrm{hn}}=4.184 \mathrm{~J}$. 
by a least-squares method. Thermodynamic functions, shown in table 2 , were derived from these smoothed heat capacities.

TABLE 2. Thermodynamic functions for strontium $\left(\mathrm{cal}_{\mathrm{th}}=4.184 \mathrm{~J}\right)$

\begin{tabular}{|c|c|c|c|c|}
\hline$\frac{T}{\mathbf{K}}$ & $\frac{C_{p}}{\mathrm{cal}_{\mathrm{th}} \mathrm{K}^{-1} \mathrm{~mol}^{-1}}$ & $\frac{S^{\circ}(T)-S^{\circ}(0)}{\mathrm{cal}_{\mathrm{th}} \mathrm{K}^{-1} \mathrm{~mol}^{-1}}$ & $\frac{H^{\circ}(T)-H^{\circ}(0)}{\mathrm{cal}_{\mathrm{th}} \mathrm{mol}^{-1}}$ & $\frac{-\left\{G^{\circ}(T)-H^{\circ}(0)\right\} / T}{\mathrm{cal}_{\mathrm{th}} \mathrm{K}^{-1} \mathrm{~mol}^{-1}}$ \\
\hline 5 & $(0.041)$ & $(0.025)$ & $(0.072)$ & $(0.010)$ \\
\hline 10 & 0.228 & 0.097 & 0.644 & 0.032 \\
\hline 15 & 0.692 & 0.268 & 2.828 & 0.079 \\
\hline 20 & 1.355 & 0.555 & 7.897 & 0.160 \\
\hline 25 & 2.059 & 0.933 & 16.441 & 0.276 \\
\hline 30 & 2.695 & 1.366 & 28.364 & 0.421 \\
\hline 35 & 3.235 & 1.823 & 43.228 & 0.588 \\
\hline 40 & 3.686 & 2.286 & 60.57 & 0.772 \\
\hline 45 & 4.056 & 2.742 & 79.95 & 0.965 \\
\hline 50 & 4.358 & 3.185 & 101.01 & 1.165 \\
\hline 60 & 4.804 & 4.022 & 146.98 & 1.573 \\
\hline 70 & 5.105 & 4.787 & 196.61 & 1.978 \\
\hline 80 & 5.320 & 5.483 & 248.80 & 2.373 \\
\hline 90 & 5.483 & 6.120 & 302.85 & 2.755 \\
\hline 100 & 5.612 & 6.704 & 358.34 & 3.121 \\
\hline 110 & 5.716 & 7.244 & 415.00 & 3.472 \\
\hline 120 & 5.802 & 7.745 & 472.61 & 3.807 \\
\hline 130 & 5.873 & 8.213 & 531.0 & 4.128 \\
\hline 140 & 5.931 & 8.650 & 590.0 & 4.436 \\
\hline 150 & 5.981 & 9.061 & 649.6 & 4.731 \\
\hline 160 & 6.023 & 9.449 & 709.6 & 5.013 \\
\hline 170 & 6.060 & 9.815 & 770.0 & 5.285 \\
\hline 180 & 6.093 & 10.162 & 830.8 & 5.547 \\
\hline 190 & 6.121 & 10.492 & 891.9 & 5.798 \\
\hline 200 & 6.147 & 10.807 & 953.2 & 6.041 \\
\hline 210 & 6.173 & 11.108 & 1014.8 & 6.275 \\
\hline 220 & 6.199 & 11.395 & 1076.7 & 6.501 \\
\hline 230 & 6.228 & 11.672 & 1138.8 & 6.720 \\
\hline 240 & 6.260 & 11.937 & 1201.3 & 6.932 \\
\hline 250 & 6.293 & 12.193 & 1264.0 & 7.137 \\
\hline 260 & 6.326 & 12.441 & 1327.1 & 7.337 \\
\hline 270 & 6.354 & 12.680 & 1390.5 & 7.530 \\
\hline 280 & 6.377 & 12.912 & 1454.2 & 7.718 \\
\hline 290 & 6.393 & 13.136 & 1518.0 & 7.901 \\
\hline 300 & 6.404 & 13.353 & 1582.0 & 8.079 \\
\hline 310 & 6.416 & 13.563 & 1646.1 & 8.253 \\
\hline 320 & 6.431 & 13.767 & 1710.4 & 8.422 \\
\hline 330 & 6.448 & 13.965 & 1774.7 & 8.587 \\
\hline 340 & 6.459 & 14.158 & 1839.3 & 8.748 \\
\hline 350 & 6.461 & 14.345 & 1903.9 & 8.905 \\
\hline 273.15 & 6.362 & 12.754 & 1410.6 & 7.590 \\
\hline 298.15 & 6.402 & 13.313 & 1570.2 & 8.047 \\
\hline
\end{tabular}




\section{Discussion}

Prior to the initiation of this study, only one report of the low-temperature heat capacity of strontium had appeared, covering only the temperature range 1.5 to $20 \mathrm{~K}^{(3)}$ A determination of the heat capacity of strontium at $300 \mathrm{~K}$ by an enthalpytype measurement was made in a water calorimeter with the sample sealed in toluene within a glass capsule. ${ }^{(4)}$ The values obtained range from 6.0 to $6.8 \mathrm{cal}_{\mathrm{th}} \mathrm{K}^{-1} \mathrm{~mol}^{-1}$ with an average value of $6.5 \mathrm{cal}_{\mathrm{th}} \mathrm{K}^{-1} \mathrm{~mol}^{-1}$. Estimates of the heat capacity and entropy of strontium have been made by Kelley ${ }^{(1)}$ and by compilers of the JANAF tables. ${ }^{(2)}$ The latter estimate of heat capacity was made by comparison of Roberts's data for strontium, calcium, and barium ${ }^{(3)}$ with other experimental data for calcium and barium. The JANAF estimate for entropy was $12.6 \mathrm{cal}_{\mathrm{th}} \mathrm{K}^{-1} \mathrm{~mol}^{-1}$ which was in reasonable accord with Kelley's estimate of $12.5 \mathrm{cal}_{\mathrm{th}} \mathrm{K}^{-1} \mathrm{~mol}^{-1}$. The JANAF compilers adopted Kelley's estimate, however, because of the large uncertainty involved in their own extrapolations.

As has been mentioned earlier, a more extensive study of the low-temperature heat capacity of strontium has appeared only recently. ${ }^{(5,6)}$ The resulting value of (13.24 \pm 0.03$) \mathrm{cal}_{\mathrm{th}} \mathrm{K}^{-1} \mathrm{~mol}^{-1}$ for the entropy at $298.15 \mathrm{~K}$ is in reasonable accord with that obtained in the present research $(13.313 \pm 0.008) \mathrm{cal}_{\mathrm{th}} \mathrm{K}^{-1} \mathrm{~mol}^{-1}$, but serious discrepancies occur between the heat capacities of the two determinations. Their heat capacities, together with those of Roberts and of Glascock, are presented in figure 1 as a deviation plot.

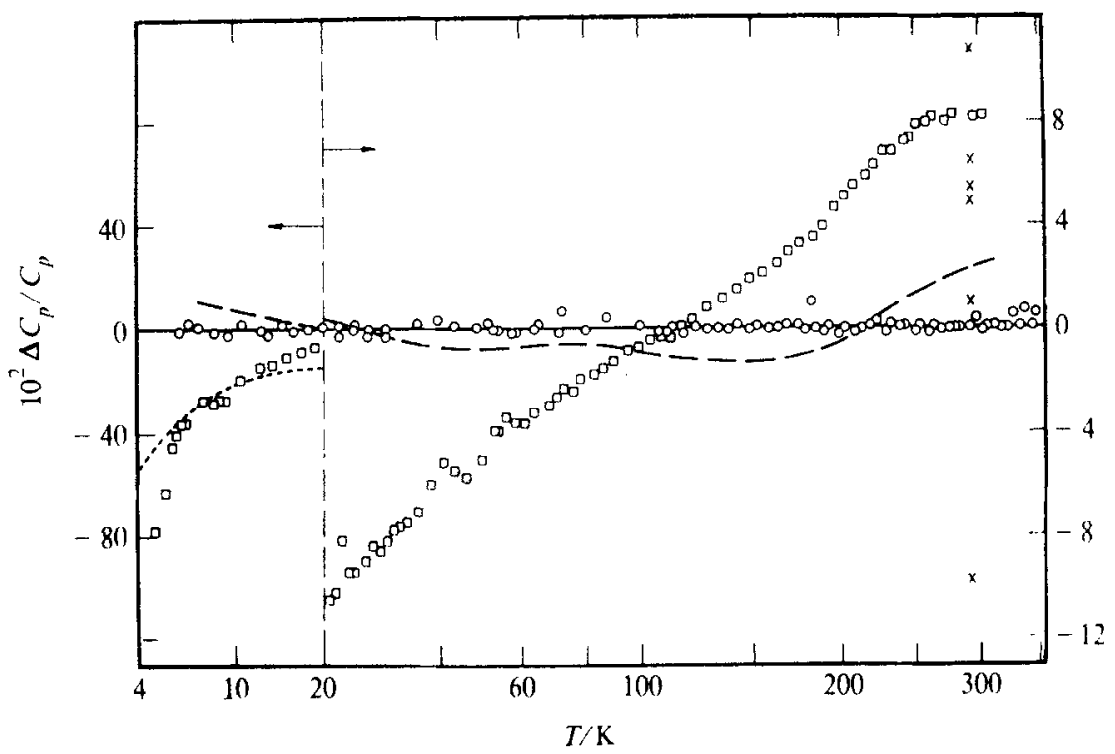

FIGURE 1. Plot of the deviation of experimental determinations from the smoothed heat-capacity curve of this study (table 2). $O$, Deviation of the experimental heat capacities of this research: $\square$, deviation of the data of Khriplovich and Paukov; $;^{(5,6)} \ldots \ldots$, data of Khriplovich and Paukov adjusted for 10 mole per cent SrO; ${ }^{(\theta)}$-...-, data of Roberts ${ }^{(3)}$ (smooth curve; author does not show original values); $x$, deviation of the data of Glascock. ${ }^{(4)}$ (Note vertical scale change at $20 \mathrm{~K}$.) 
In view of the high reactivity of strontium with oxygen and water, the presence of strontium monoxide in a sample of strontium metal must be considered. The analytical results on our sample indicated that the oxygen content was negligible. Therefore, in an attempt to explain the systematic differences between our values and those of Khriplovich and Paukov ${ }^{(5,6)}$ in the absence of complete analytical results on their sample, we arbitrarily assumed 10 moles per cent strontium monoxide to be present in their sample. Adjustment of their heat capacity for the presence of strontium monoxide was made using the data of Anderson ${ }^{(9)}$ over the temperature range 57 to $229 \mathrm{~K}$, and at lower temperatures, by extrapolation of the curve of Debye, $\theta$ against $T$. The adjustment greatly reduces the deviation from our smooth curve, as shown in figure 1.

This, of course, does not prove the sample impure. Khriplovich and Paukov reported mass percentages of major metallic impurities: $0.01 \mathrm{Cr}, 0.03 \mathrm{Fe}, 0.5 \mathrm{~V}, 0.1 \mathrm{Mg}$. Elements not detected, but with rather high limits of detectability, were $\mathrm{Si}$ and $\mathrm{Sb}$, with limits of 0.03 and 0.01 , respectively. However, a portion of the material understood to be from the same stock as that used by the investigators at Novosibirsk was analyzed in this country and was found to contain $(6 \pm 3)$ moles per cent of oxygen. ${ }^{(10)}$ Another portion of the same stock was shown in Moscow to contain approximately the same level of oxygen impurity. ${ }^{(11)}$ These figures are many times higher than those claimed by the authors ${ }^{(6,12)}$ and suggest, at least, that a re-examination of the purity of that sample be made. In the meantime, we consider our heat capacities to be more reliable.

We have also compared our values of $\alpha$ and $\beta$ obtained in the plot of $C_{p} / T$ against $T^{2}$ with those obtained by Roberts ${ }^{(3)}$ and by Khriplovich and Paukov. ${ }^{(6)}$ The values in the present study $\left(\alpha=1.99 \times 10^{-4} \mathrm{cal}_{\mathrm{th}} \mathrm{K}^{-4} \mathrm{~mol}^{-1}\right.$ and $\beta=3.3 \times 10^{-3} \mathrm{cal}_{\mathrm{th}} \mathrm{K}^{-2}$ $\left.\mathrm{mol}^{-1}\right)$ differ significantly from those of Roberts $\left(\alpha=1.46 \times 10^{-4} \mathrm{cal}_{\mathrm{th}} \mathrm{K}^{-4} \mathrm{~mol}^{-1}\right.$, $\left.\beta=8.6 \times 10^{-4} \mathrm{cal}_{\mathrm{th}} \mathrm{K}^{-2} \mathrm{~mol}^{-1}\right) ; \beta$ is thus seen to be much closer to the free-electron model value and possibly indicates less overlap-than found earlier-of the first two Brillouin zones. ${ }^{(3)}$ It is realized that temperatures of 5 to $10 \mathrm{~K}$ are rather high for precise determination of $\alpha$ and $\beta$ for a metal. However, Roberts's sample prepared by a commercial supplier contained 2 per cent of metallic impurities and ". . . most of the surface oxide was removed in the air ...".(3) The plot of $C_{p} / T$ against $T^{2}$ of the presumably oxide-rich sample of Khriplovich and Paukov ${ }^{(5)}$ below $10 \mathrm{~K}$ is comparable to that of Roberts.

It is interesting to note that the plot of standard entropies of the alkaline earth metals $(\mathrm{Mg}, \mathrm{Ca}, \mathrm{Sr}$, and $\mathrm{Ba}$ ) against the logarithm of the molar mass is essentially linear, provided slight adjustments for crystal-structure differences are made by Gschneider's method. ${ }^{(13)}$

The high-temperature heat capacity of the identical sample is being explored at the Sandia Laboratories, Albuquerque, New Mexico, by $\mathrm{Dr}$ Howard Stephens. His results on a portion of the same sample of material ${ }^{(14)}$ are consistent with the trend of our values at the upper limit of our temperature range.

We express appreciation to Dr David T. Peterson of Iowa State University for his preparation of a high-purity sample and supervision of some of the analytical work 
and to Albert Highe for experimental measurements and calculations on the thermophysical properties. Moreover, we express our appreciation to Dr Charles E. Holley of the Los Alamos Scientific Laboratory for his leadership and arrangement for the procurement of the samples and the sharing of them with the other laboratories involved. In addition, he, together with Professor Irwin J. Brink of Hope College, determined the enthalpy of solution of a portion of the same sample of metal to obtain the enthalpy of formation of strontium monoxide.

\section{REFERENCES}

1. Kelley, K. K.; King, E. G. U.S. Bur. Mines Bull. 1961, No. 592.

2. JANAF Thermochemical Tables, The Dow Chemical Company, Midland, Michigan, Dec. 31, 1970.

3. Roberts, L. M. Proc. Phys. Soc. London 1957, B70, 738.

4. Glascock, B. L. J. Am. Chem. Soc. 1910, 32, 1222.

5. Khriplovich, L. M.; Paukov, I. E. Zhur. Fiz. Khim. 1975, 567 (extended abstract only).

6. Khriplovich, L. M.; Paukov, I. E. VINITI Documentary Deposit No. 3190-75, 11 November, 1975.

7. Peterson, D. T.; Fattore, V. G. Anal. Chem. 1962, 34, 579.

8. Westrum, E. F., Jr.; Furukawa, G. T.; McCullough, J. P. In Experimental Thermodynamics, Vol. 1, p. 133, McCullough, J. P.; Scott, D. W.; editors. Butterworths: London. 1968.

9. Anderson, C. T. J. Am. Chem. Soc. 1935, 57, 429.

10. Reported by Holley, C. E. personal communication, July 1976.

11. Reported by Gurvich, L. V. personal communication, August 1976.

12. Paukov, I. E. personal communication, October 1976.

13. Gschneider, K. J. Less Common Met. 1975, 43, 179.

14. Stephens, H. P. personal communication, February 1977. 